\title{
THE STRUCTURE OF A FINITELY GENERATED KLEINIAN GROUP
}

\author{
BY \\ LARS V. AHLFORS \\ Harvard University, Cambridge, Mass., U.S.A.( $\left.{ }^{1}\right)$
}

In his classical work on automorphic functions Poincaré introduced the distinction between Fuchsian groups and the more general class of Kleinian groups. The Fuchsian case proved to be much more tractable, and soon grew to an impressive theory with applications in several branches of mathematics. In contrast, the study of Kleinian groups progressed slowly and still presents major unsolved problems. A recent surge of interest occurred when it was found that the theory of quasiconformal mappings has important bearing on the subject.

The writer proved (Ahlfors [1]), with minor restrictions that were subsequently removed by Greenberg [7] and Bers [5], that the orbit space of a finitely generated Kleinian group, when completed, is a finite union of compact Riemann surfaces. This result is the starting point of newer developments.

An important step was taken by Bers who, in several papers $[3,4,5]$, emphasized the use of differentials of arbitrary order $q \geqslant 2$. This served to eliminate the deficiencies of the original approach, which, motivated by the connection with quasiconformal mappings, worked exclusively with quadratic differentials.

This paper continues the research of Bers, especially as developed in [4]. The reader may wish to consult Ahlfors [2] for notations, which differ from those of Bers, and for an assembly of elementary facts. The present paper is, however, essentially selfcontained.

The paper of Bers, at least indirectly, and my own, very directly, owe much to Eichler [6]. I would be remiss if I did not acknowledge my debt accrued by rereading his paper. I have also profited greatly from Weil [8].

(1) This research was partially supported by the Air Force Office of Scientific Research under Contract AF 49(638)-1951.

1-692905 Acta mathematica 122. Imprimé le 18 mars 1969 


\section{Differentials}

1.1. In this paper $\Gamma$ denotes a finitely generated Kleinian group. By definition, a Kleinian group is a group of fractional linear transformations of the extended complex plane whose set of limit points is a nowhere dense perfect set. It is important to notice that we are excluding the elementary groups, which are the ones with at most two limit points.

The complement of the limit point set is the set of discontinuity, to be denoted by $\Omega=\Omega(\Gamma)$. As already mentioned, the orbit space $S=S(\Gamma)=\Omega / \Gamma$ is known to be a finite union of Riemann surfaces $S_{i}$, each obtained from a compact surface $\bar{S}_{i}$ by removing at most a finite number of points. We shall also use the notation $\bar{S}=\Sigma \bar{S}_{i}$.

The inverse image of $S_{i}$ under the projection map $\Omega \rightarrow \Omega / \Gamma$ will be denoted by $\Omega_{i}$, its components by $\Omega_{i j}$. The projection defines each $\Omega_{i j}$ as a branched covering of $S_{i}$ with ramification numbers $r\left(p_{k}\right)>1$ at the projections of elliptic fixed points. We set $r\left(p_{k}\right)=\infty$ when $p_{k} \in \bar{S}_{i}-S_{i}$ and refer to these points as infinite branch points or cusps.

1.2. A meromorphic function $\varphi$ on $\Omega$ is called a differential of order $q$ with respect to $\Gamma$ if (i) $\varphi(A z) A^{\prime}(z)^{q}=\varphi(z)$ for all $A \in \Gamma$, (ii) the projection of $\varphi$ can be extended to a rational differential on $S$. More precisely, $\phi$ is the projection of $\varphi$ on $S$ if $\phi(Z) d Z^{q}=\varphi(z) d z^{\alpha}$ becomes an identity when $Z$ is replaced by the local coordinate of the projection of $z$. It follows from (i) that $\phi$ is invariantly defined, and (ii) requires that $\phi$ can be extended to $S$ with no singularities other than poles.

The linear space formed by all differentials of order $q$ will be denoted by $D^{q}=D^{a}(\Gamma)$. There is an obvious direct sum decomposition $D^{q}=\sum D_{i}^{q}$ where $\varphi \in D_{i}^{q}$ vanishes identically outside of $\Omega_{i}$; usually, we identify such a $\varphi$ with its restriction to $\Omega_{i}$.

1.3. At unramified points the degrees (orders) of $\varphi$ and $\phi$ are equal. For a point with finite ramification number $r=r\left(p_{k}\right)>1$ we may assume that the projection is given by $Z=z^{r}$. If $\varphi$ and $\phi$ have degrees $v$ and $\tilde{v}$ respectively, one obtains $r \tilde{v}+q(r-1)=v$. It follows that $\varphi$ is regular if and only if $\tilde{v} \geqslant \tilde{v}_{0}=-[q(1-1 / r)]$; here $[x]$ is the largest integer $\leqslant x$. For this reason, it is natural to define the reduced degree of $\varphi$ as the number $\tilde{v}-\tilde{\nu}_{0}$. For instance, we would say that $\varphi$ has a simple pole if the reduced degree is -1 . We note that $\tilde{v}_{0}=0$ when $q=1$ or 0 .

A cusp corresponds to a certain subgroup of parabolic transformations with generator $B \in \Gamma$. For the present purpose $\Gamma$ can be replaced by any conjugate group, and it is therefore no restriction to assume that $B z=z+1$. In these circumstances $\Omega_{i}$ contains a half plane $\operatorname{Im} z>$ const. (for a proof see Ahlfors [1]), and we may choose $Z=e^{2 \pi i z}$ as local variable. The relation between $\varphi$ and $\phi$ is given by $(2 \pi i)^{q} \phi(Z) Z^{q}=\varphi(z)$, and $\varphi$ has period 1 . 
The regularity of $\varphi$ is expressed by the condition $\varphi(z)=O\left(|z|^{-2 q}\right)$ as $\operatorname{Im} z \rightarrow+\infty$. For $q>0$ this will be satisfied whenever $\tilde{v}=\operatorname{deg} \phi \geqslant 1-q$, whereas for $q \leqslant 0$ the condition becomes $\tilde{\nu} \geqslant-q$. Accordingly, we set $\tilde{\nu}_{0}=1-q$ if $q>0, \tilde{v}_{0}=-q$ if $q \leqslant 0$. Observe that $\tilde{v}_{0}$ is again 0 for $q=1$ and $q=0$. It may also be noted that $\tilde{\nu}_{0}$ is always the limit of $-[q(1-1 / r)]$ as $r \rightarrow \infty$. The reduced degree of $\varphi$ is again defined as $\tilde{\boldsymbol{v}}-\tilde{y}_{0}$.

The Fourier development of a regular $\varphi$ begins with a positive power of $Z$ if $q>0$, but when $q \leqslant 0$ there may be a constant term. Whether $\varphi$ is regular or not, the constant term plays a different role from the others. To bring out this distinction we shall say that a differential $\varphi$ is parabolic if, at each cusp, the constant term of the Fourier development is missing. For differentials in $D_{i}^{q}$ only the cusps on $\bar{S}_{i}$ are relevant,

1.4. Let $\alpha$ be a divisor on $\bar{S}$. We shall say that $\varphi \in D^{q}$ is a multiple of $\alpha$ if, at every point, the reduced degree of $\varphi$ is at least equal to the coefficient of $\alpha$. The linear space of multiples of $\alpha$ is denoted by $D^{\alpha}(\alpha)$. In particular, the space of everywhere regular differentials, also known as cusp forms, is denoted by $D^{q}(0)$. The meaning of the notations $D_{i}^{q}(\alpha), D_{i}^{q}(0)$ is obvious.

The Riemann-Roch theorem leads to a relation between $\operatorname{dim} D_{i}^{q}(\alpha)$ and $\operatorname{dim}$ $D_{i}^{1-a}(-\alpha)$. If $g_{i}$ is the genus of $\bar{S}_{i}$ this relation reads

$$
\operatorname{dim} D_{i}^{q}(\alpha)=\operatorname{dim} D_{i}^{1-q}(-\alpha)-\sum_{p \in \bar{S}_{i}} \tilde{\nu}_{0}(p)+(2 q-1)\left(g_{i}-1\right)-\operatorname{deg} \alpha .
$$

Because $\Gamma$ has more than two limit points the ramification numbers have to satisfy the condition

$$
2 g_{i}-2+\sum_{p \in \bar{S}_{i}}(1-1 / r(p))>0
$$

which expresses the fact that the Poincaré area of $S_{i}$ is positive. It is an easy and wellknown consequence that $D_{i}^{q}(0)=0$ whenever $q<0$.

From now on we assume that $q \geqslant 2$. If we apply (1) with $\alpha=0$ we obtain

$$
\operatorname{dim} D_{i}^{q}(0)=-\sum_{p \in \bar{S}_{i}} \tilde{\nu}_{0}(p)+(2 q-1)\left(g_{i}-1\right)
$$

and it follows that (1) can be written in the convenient form

$$
\operatorname{dim} D_{i}^{q}(\alpha)=\operatorname{dim} D_{i}^{1-q}(-\alpha)+\operatorname{dim} D_{i}^{q}(0)-\operatorname{deg} \alpha .
$$

\section{Cohomology and Eichler integrals}

2.1. We let $A \in \Gamma$ act on the space of functions $f: \Omega \rightarrow \mathrm{C}$ according to the rule $(f \cdot A)(z)=$ $f(A z) A^{\prime}(z)^{1-q}$. Since $f \cdot A B=(f \cdot A) \cdot B$ this defines a representation of $\Gamma$ in the space of linear automorphisms of $\Omega^{\mathrm{c}}$. 
In particular, $\Gamma$ acts on the space $V$ of polynomials of degree at most $2 q-2$, and it leaves $V$ invariant. There is thus a canonical representation of $\Gamma$ in the space of linear autom orphisms of $\mathbf{C}^{2 \boldsymbol{q}-\mathbf{1}}$.

A cocycle on $\Gamma$ is a mapping $h: \Gamma \rightarrow V$ such that $h(A B)=h(A) \cdot B+h(B)$. It is a coboundary if there exists a $v \in V$ with $h(A)=v \cdot A-v$ for all $A \in \Gamma$. The cocycles modulo coboundaries form the first cohomology group $H^{1}(\Gamma, V)$. We shall simplify the notation to $H(\Gamma)$, and we remind the reader that it depends on the integer $q \geqslant 2$.

2.2. A meromorphic function $f$ on $\Omega_{i}$ will be called an Eichler integral on $\Omega_{i}$ if

(i) $\partial^{2 q-1} f \in D_{q}^{i}$

(ii) $f \cdot A-f \in V$ for all $A \in \Gamma$.

Here $\partial$ means differentiation with respect to $z$. It is a fundamental fact that $\partial^{2 q-1}(f \cdot A)=$ $\left[\left(\partial^{2 q-1}\right) \cdot A\right] A^{\prime}(z)^{q}$. It is therefore a consequence of (i) that $f \cdot A-f$ is a polynomial of degree at most $2 q-2$ in each component of $\Omega_{i}$. Condition (ii) states that this polynomial does not change from component to component.

The space of all Eichler integrals on $\Omega_{i}$ will be denoted by $E_{i}=E_{i}(\Gamma)$. We shall also need to consider various subspaces. An Eichler integral $f$ will be called parabolic if $\partial^{2 q-1} f$ is parabolic (see 1.3), and the parabolic subspace is denoted by $P E_{i}$. An integral is said to be of the first kind, and we write $f \in E_{i 1}$, if $\partial^{2 q-1} f \in D_{i}^{q}(0)$. Finally, $E_{i 0}$ will be the space of trivial Eichler integrals, characterized by $\partial^{2^{q-1}} f=0$. A trivial integral is represented by a polynomial on each component $\Omega_{i j}$, but these polynomials are not arbitrary, for they are still subject to condition (ii).

2.2. The polynomials $f \cdot A-f$ are the periods of $f$, and we write $f \cdot A-f=\operatorname{pd}_{A} f$. It is readily verified that the mapping $A \rightarrow \operatorname{pd}_{A} f$ is a cocycle. The periods determine a canonical homomorphism $E_{i} \rightarrow H(\Gamma)$ which will be referred to as the period homomorphism. We denote it by pd; thus pd $f$ is the cohomology class of the periods of $f$, and $\operatorname{pd} E_{i}$ is the image of $E_{i}$ under the period mapping.

The periods of parabolic Eichler integrals have a special property. We have seen that a cusp determines a parabolic transformation $B \in \Gamma$, unique up to conjugation. A cocycle is said to be parabolic at the cusp corresponding to $B$ if $h(B)=v \cdot B-v$ for some $v \in V$. To make the definition consistent we must show that this property is preserved under conjugation.

Quite generally, let us replace $\Gamma$ by $\Gamma^{U}=U \Gamma U^{-1}$ where $U$ is an arbitrary linear transformation, not necessarily in $\Gamma$. For a cocycle $h$ on $\Gamma$ we define $h^{U}$ on $\Gamma^{U}$ by $h^{U}\left(U A U^{-1}\right)=$ $h(A) \cdot U^{-1}$. We check first that $h^{U}$ is a cocycle. One obtains, indeed, $h^{U}\left(U A U^{-1} U B U^{-1}\right)=$ 
$h(A) \cdot B U^{-1}+h(B) \cdot U^{-1}=h^{U}\left(U A U^{-1}\right) \cdot U B U^{-1}+h^{U}\left(U B U^{-1}\right)$. Next, if $h(B)=v \cdot B-v$ we find $h^{U}\left(U B U^{-1}\right)=v \cdot B U^{-1}-v \cdot U^{-1}=\left(v \cdot U^{-1}\right) \cdot U B U^{-1}-v \cdot U^{-1}$, and the invariance of the parabolicity condition is proved. The same computation shows that $h^{v}$ is a coboundary if $h$ is a coboundary. We have thus defined a canonical isomorphism between $H(\Gamma)$ and $H\left(\Gamma^{U}\right)$.

We may now say that a cocycle is parabolic on $\Omega_{i}$ if it satsfies the parabolicity condition for all cusps on $\bar{S}_{i}$. Since coboundaries are automatically parabolic we may speak of cohomology classes being parabolic on $\bar{S}_{i}$, and the space of such cohomology classes will be denoted by $P_{i} H(\Gamma)$.

LEM A 1. The period of a parabolic integral is parabolic.

Proof. We claim, more precisely, that $f \in P E_{i}$ implies pd $f \in P_{i} H(\Gamma)$. Our preliminary considerations have shown that we may take the transformation $B$ that corresponds to a given cusp to be of the form $B z=z+1$. Let $\Omega_{i j}$ be the component that contains an upper half plane. In that half plane $\varphi=\partial^{2 q_{-1}} f$ has a convergent development $\varphi=\sum c_{m} e^{2 \pi i m z}$ with $c_{0}=0$. It follows by iterated integration inside the half plane that $f$ is itself a periodic function plus a polynomial $v$ of degree $\leqslant 2 q-2$. Hence the period of $f$ is $v(z+1)-v(z)=$ $v \cdot B-v$, and the parabolicity condition is fulfilled.

\section{Summary of results}

3.1. Because $\Gamma$ is finitely generated the cohomology group $H(\Gamma)$ has finite dimension. In fact, if there are $N$ generators it is easy to see that $\operatorname{dim} H(\Gamma)$ cannot exceed $(N-1)(2 q-1)$. It is natural to ask to what extent the cohomology classes are the periods of Eichler integrals belonging to $E_{i}$ and its subspaces. In other words, we are interested in information that describes the images $\operatorname{pd} E_{i}, \operatorname{pd} P E_{i}, \operatorname{pd} E_{i 1}, \operatorname{pd} E_{i 0}$. The main result is contained in two theorems.

THEOREM 1. $\operatorname{dim}\left(\operatorname{pd} P E_{i} / \operatorname{pd} E_{i 1}\right)=\operatorname{dim} D_{i}^{q}(0)$.

THEOREM 2. $\operatorname{pd} E_{i}=H(\Gamma)$ and $\operatorname{pd} P E_{i}=P_{i} H(\Gamma)$.

The second theorem asserts that the period homomorphisms $E_{i} \rightarrow H(\Gamma)$ and $P E_{i} \rightarrow$ $P_{i} H(\Gamma)$ are surjective. In other words, every cohomology is a period of an Eichler integral on $\Omega_{i}$, and every parabolic cohomology comes from a parabolic integral. The first theorem, perhaps even more informative, lets us recognize, in terms of a known quantity associated with $\bar{S}_{i}$, the size of the contribution from essentially different parabolic integrals of the second kind.

3.2. We shall write $d_{i}=\operatorname{dim} D_{i}^{q}(0)$, and we recall that this dimension is given by formula (1). In order to analyze the theorems we shall also introduce the dimensions 
$p_{i}=\operatorname{dim}\left(\operatorname{pd} E_{i} / \operatorname{pd} P E_{i}\right), e_{i}=\operatorname{dim}\left(\operatorname{pd} E_{i 1} / \operatorname{pd} E_{i 0}\right), s_{i}=\operatorname{dim} \operatorname{pd} E_{i 0}$. We shall also denote the number of cusps by $n_{i}$.

The theorems express a balance

$$
p_{i}+d_{i}+e_{i}+s_{i}=\operatorname{dim} H(\Gamma)
$$

between the various dimensions. The value of the information depends on the extent to which we can characterize the numbers involved in other ways.

The strongest result is the characterization of $d_{i}$ through Theorem 1 . As for $p_{i}$, Theorem 2 shows that $p_{i}=\operatorname{dim} H(\Gamma) / P_{i} H(\Gamma)$. The parabolicity introduces one linear condition for each eusp, and we deduce that $p_{i} \leqslant n_{i}$. The fact that $D_{i}^{1-a}(0)=0$ shows that the period homomorphism, restricted to $E_{i 1}$, has kernel $V$. It follows that $e_{i}=\operatorname{dim}\left(E_{i 1} / E_{i 0}\right)$, and we may interpret $e_{i}$ as the number of essentially different integrals of the first kind. The mapping $\partial^{2 q-1}: E_{i 1} \rightarrow D_{i}^{q}(0)$ has kernel $E_{i 0}$, proving that $e_{i} \leqslant d_{i}$. Finally, $s_{i}$ indicates the number of ways in which 0 on one of the components $\Omega_{i j}$ can be extended to a trivial integral. It is zero when $\Omega_{i}$ is connected.

In a general way, if $S_{i}$ has low genus and ramification, the number $s_{i}$ must be large. There seems to be no intuitive interpretation of $s_{i}$ beyond saying that $s_{i}$ is large when the components $\Omega_{i j}$ are strongly permuted by $\Gamma$.

3.3. Let $I$ be any set of the subscripts $i$. We shall let $\Omega_{I}$ be the union of the corresponding $\Omega_{i}$. An Eichler integral on $\Omega_{I}$ will be defined as one that has the same periods on all $\Omega_{i}$ with $i \in I$. The notations $E_{I}, P E_{I}, E_{11}, E_{10}$ and $P_{I} H(\Gamma)$ are selfexplanatory. We are going to show that Theorem 1 can be generalized to this situation.

THEOREM 3. $\operatorname{dim}\left(\operatorname{pd} P E_{I} / \operatorname{pd} E_{I 1}\right)=\operatorname{dim} D_{I}^{q}(0)$.

Here, naturally, $\operatorname{dim} D_{I}^{q}(0)=d_{I}=\sum_{i \in I} d_{i}$. There is also a counterpart of Theorem 2.

Theorem 4. pd $E_{I}=H(\Gamma)$ and pd $P E_{I}=P_{I} H(\Gamma)$.

This happens to be a trivial consequence of Theorem 2, for it suffices to apply that theorem separately to each $\Omega_{i}$. It becomes important, nevertheless, when used in combination with Theorem 3 . In analogy with (5) we obtain, with obvious notations,

$$
p_{I}+d_{I}+e_{I}+s_{I}=\operatorname{dim} H(\Gamma) \text {. }
$$

For instance, we conclude at once that $\operatorname{dim} D_{q}(0)=\sum d_{i} \leqslant \operatorname{dim} H(\Gamma)$, a theorem due to Bers [4]. 
Combining (5) and (6) yields

$$
p_{i}+d_{i}+e_{i}+s_{i}=p_{I}+d_{I}+e_{I}+s_{I},
$$

a piece of information that no longer involves cohomology. Consider the special case where $\Omega_{i}$ is connected, so that $s_{i}=0$, and take $I$ to be the set of all indices. Since $p_{i} \leqslant p_{I}$ one obtains $d_{I}+e_{I} \leqslant d_{i}+e_{i} \leqslant 2 d_{i}$. This contains Bers' second theorem, $d_{I} \leqslant 2 d_{i}$, from the same paper.

\section{Subgroups and restrictions}

4.1. Recall that we are denoting the components of $\Omega_{i}$ by $\Omega_{i j}$. Let $\Gamma_{i j}$ be the subgroup of $\Gamma$ that leaves $\Omega_{i j}$ invariant. For fixed $i$, the different $\Omega_{i k}$ are in one to one correspondence with the right cosets of $\Gamma_{i j}$. If $\Omega_{i k}=B \Omega_{i j}$, then $\Gamma_{i k}=B \Gamma_{i j} B^{-1}$, and $\Gamma_{i k}=\Gamma_{i j}$ if and only if $B$ is in the normalizer of $\Gamma_{i j}$.

LEMMA 2. $\Gamma_{i j}$ is finitely generated, and $\Omega_{i j}$ is a component of $\Omega\left(\Gamma_{i j}\right)$. In particular, $\Gamma_{i j}$ is a Kleinian group.

Proof. We remove the elliptic fixed points from $\Omega_{i j}$ and denote the punctured region by $\Omega_{i j}^{0}$, its projection by $S_{i}^{0}$. In familiar manner, $\Omega_{i j}^{0}$ is a smooth covering surface of $S_{i}^{0}$ determined by a normal subgroup $N$ of the fundamental group $\pi_{1}\left(S_{i}^{0}\right)$ and $\Gamma_{i j}$ is isomorphic to the quotient group $\pi_{1}\left(S_{i}^{0}\right) / N$. Since $\pi_{1}\left(S_{i}^{0}\right)$ is finitely generated, so is the quotient group, and consequently $\Gamma_{i j}$. We conclude that $S\left(\Gamma_{i j}\right)$ can be completed to a finite union $\bar{S}\left(\Gamma_{i j}\right)$ of compact Riemann surfaces; this would be true even if $\Gamma_{i j}$ were an elementary group.

Every orbit of $\Gamma$ in $\Omega_{i}$ contains a unique orbit of $\Gamma_{i j}$ in $\Omega_{i j}$. This determines an injective conformal mapping of $S_{i}$ into $S\left(\Gamma_{i j}\right)$, and hence into $\bar{S}\left(\Gamma_{i j}\right)$. As $p \in S_{i}$ approaches a cusp, so does its image, for the parabolic transformation $B$ determined by the cusp on $S_{i}$ belongs to $\Gamma_{i j}$ and defines a cusp on $\bar{S}\left(\Gamma_{i j}\right)$ with the same local coordinate system. We conclude that the mapping extends to a continuous and open map from $\bar{S}_{i}$ to $\bar{S}\left(\Gamma_{i j}\right)$. The image must be a full component, and it follows that the component of $\Omega\left(\Gamma_{i j}\right)$ which contains $\Omega_{i j}$ cannot contain points not in $\Omega_{i j}$. The components coincide and since $\Omega_{i j}$ has more than two boundary points, $\Gamma_{i j}$ is Kleinian.

In another terminology, $\Gamma_{i j}$ is of the first kind in $\Omega_{i j}$.

4.2. A cocycle on $\Gamma$ can be restricted to a cocycle on $\Gamma_{i j}$, and the restriction of a coboundary is a coboundary. We can therefore speak of the restriction $h \mid \Gamma_{i j}$ of any $h \in H(\Gamma)$, and of the space $H(\Gamma) \mid \Gamma_{i j}$ as a subspace of $H\left(\Gamma_{i j}\right)$.

It is clear what we mean by an Eichler integral with respect to $\Gamma_{i j}$, considered on $\Omega_{i j}$. An integral with respect to $\Gamma$ defines, by restriction, an integral on $\Omega_{i j}$, but it is not 
sure that an integral on $\Omega_{i j}$ can be extended to an integral on $\Omega_{i}$. The following simple lemma is therefore important.

LEMMA 3. If an Eichler integral on $\Omega_{i j}$ has the period $h \mid \Gamma_{i j}$ for some $h \in H(\Gamma)$, then it can be extended to an Eichler integral on $\Omega_{\mathfrak{i}}$ with period $h$.

Proof. If $f$ is the given integral on $\Omega_{i j}$ we define its extension $\hat{f}$ by setting $\hat{f}=f \cdot A^{-1}-$ $h\left(A^{-1}\right)$ on $A \Omega_{i j}$. This definition is independent of the choice of $A$ from the right coset of $\Gamma_{i j}$ to which it belongs, for if $A \Omega_{i j}=B \Omega_{i j}$, then $B^{-1} A \in \Gamma_{i j}$ and $f \cdot B^{-1} A-f=h\left(B^{-1} A\right)$ on $\Omega_{i j}$, hence $f \cdot B^{-1}-f \cdot A^{-1}=h\left(B^{-1} A\right) \cdot A^{-1}=h\left(B^{-1}\right)+h(A) \cdot A^{-1}=h\left(B^{-1}\right)-h\left(A^{-1}\right)$ on $A \Omega_{i j}$.

To show that $\hat{f}$ is an Eichler integral we compute the period $\operatorname{pd}_{B} \hat{f}$ on $A \Omega_{i j}$ for an arbitrary $B \in \Gamma$. For $z \in A \Omega_{i j}, f(B z)=\left(f \cdot A^{-1} B^{-1}\right)(B z)-h\left(A^{-1} B^{-1}\right)(B z)$ and hence $f \cdot B=$ $f \cdot A^{-1}-h\left(A^{-1} B^{-1}\right) \cdot B=f \cdot A^{-1}-h\left(A^{-1}\right)+h(B)$. Thus $\operatorname{pd}_{B} \hat{f}=h(B)$, independently of $A$, and we conclude that $f \in E_{i}$.

COROLLARY. The image pd $E_{i 0}$ consists of all $h \in H(\Gamma)$ with $h \mid \Gamma_{i j}=0$, and

$$
H(\Gamma) / p d E_{i 0}=H(\Gamma) \mid \Gamma_{i j} \text {. }
$$

This is the special case of the lemma where $f=0$ on $\Omega_{i j}$.

\section{The cohomology group $H\left(\pi_{i}\right)$}

5.1. We return to the punctured surface $S_{i}^{0}$ and its fundamental group $\pi_{1}\left(S_{i}^{0}\right)$ considered in Section 4.1. We assume that the fundamental group is formed with respect to an initial point $p_{0}$, and we choose simple loops $c_{k}$ from $p_{0}$ around the branch points $p_{k}$ of finite order $r_{k}$. Let $N^{\prime}$ be the smallest normal subgroup of $\pi_{1}\left(S_{i}^{0}\right)$ that contains the powers $c_{k}^{r}$, and set $\pi_{i}=\pi_{1}\left(S_{i}^{0}\right) / N^{\prime}$. Clearly, $N^{\prime}$ is a subgroup of $N$, the group determined by $\Omega_{i j}$ as a covering of $S_{i}^{0}$, and it determines a surface $\tilde{S}_{i}$ which may be viewed as a covering of $\Omega_{i j}$. Although $\pi_{i}$ can be realized as a Fuchsian group, we shall actually have no need for this concrete realization.

Choose $z_{0} \in \Omega_{i}$, with projection $p_{0}$. A closed curve $c$ from $p_{0}$ lifts to a curve $c^{\prime}$ on $\Omega_{i j}$ that leads from $z_{0}$ to a point $A z_{0}$, where $A$ is a uniquely determined element $\sigma(c) \in \Gamma_{i j}$. Since $\sigma(c)$ is the identity when $c \in N^{\prime}$, this construction defines a surjective homomorphism $\sigma: \pi_{i} \rightarrow \Gamma_{i j}$.

We define the action of $\pi_{i}$ on functions in $\Omega_{i j}$ by $f \cdot c=f \cdot \sigma(c)$. The cohomology group defined by this action is denoted by $H\left(\pi_{i}\right)$, and the subgroup of parabolic elements by $P H\left(\pi_{i}\right)$. 
LEMMA 4. $\operatorname{dim} P H\left(\pi_{i}\right)=2 d_{i}$ and $\operatorname{dim} H\left(\pi_{i}\right)=2 d_{i}+n_{i}$.

We recall that $d_{i}=\operatorname{dim} D_{i}^{q}(0)$ and that $n_{i}$ is the number of cusps on $S_{i}$. Proofs are given in Eichler [6] and Weil [8]. Eichler treats a less general and Weil a more general case. In the interest of completeness we shall reproduce Weil's proof with appropriate modifications.

5.2. Proof of Lemma 4. We represent $\pi_{i}$ through generators $a_{j}, b_{j}, c_{k}$ subject to the relations $w=a_{1} b_{1} a_{1}^{-1} b_{1}^{-1} \ldots a_{g} b_{g} a_{g}^{-1} b_{g}^{-1} c_{1} \ldots c_{n}=1$ and $c_{k}^{r_{k}=1}$ for the loops that correspond to finite branch points. We have to determine the number of linearly independent cocycles and parabolic cocycles on the free group that satisfy $h(w)=0$ and $h\left(c_{c}^{r_{k}}\right)=0$.

The latter condition can be written as $h\left(c_{k}\right) \cdot\left(c_{k}^{r_{k}-1}+\ldots+c_{k}+1\right)=0$ in readily understandable notation. Let $V_{k}$ be the space of all $v \in V$ that satisfy $v \cdot\left(c_{k}^{r_{k}-1}+\ldots+1\right)=0$. For the purpose of finding the dimension of $V_{k}$ we may assume that $\sigma\left(c_{k}\right)$ is the mapping $z \rightarrow \omega z$ with $\omega^{r_{k}}=1$. Then $z^{m} \cdot\left(c_{k}^{r_{k}-1}+\ldots+1\right)=\left(\omega^{\left(r_{k}-1\right)(m+1-q)}+\ldots+\omega^{m+1-q}+1\right) z^{m}$, and this fails to be zero only if $r_{k}$ divides $m+1-q$. In the interval $0 \leqslant m \leqslant 2 q-2$ this happens $2\left[(q-1) / r_{k}\right]+1$ times. We conclude that

$$
\operatorname{dim} V_{k}=2 q-1-2\left[(q-1) / r_{k}\right]-1=-2\left[(1-q)\left(1-1 / r_{k}\right)\right]
$$

Simple estimates show that $[q(1-1 / r)]+[(1-q)(1-1 / r)]=0$. On comparison with the definition of $\tilde{v}_{0}$ in Section 1.3 it follows that $\operatorname{dim} V_{k}=-2 \tilde{v}_{0}\left(p_{k}\right)$.

We notice further that $V_{k}=V \cdot\left(c_{k}-1\right)$. Indeed, if $v=u \cdot c_{k}-u$ one verifies that

$$
v\left(c_{k}^{r_{k}-1}+\ldots+1\right)=0
$$

and if this condition is fulfilled we choose

$$
u=v \cdot\left(c_{k}^{r_{k}-1}+2 c_{k}^{r_{k}-2}+\ldots+\left(r_{k}-2\right) c_{k}+r_{k}-1\right)
$$

and obtain $u \cdot c_{k}-u=-\left(r_{k}-1\right) v$.

When $r_{k}=\infty$ we let $V_{k}$ be the space of polynomials that satisfy the parabolicity condition $v=u \cdot c_{k}-u, u \in V$; in other words, $V_{k}=V \cdot\left(c_{k}-1\right)$ by definition. In order to find $\operatorname{dim} V_{k}$ we may take $\sigma\left(c_{k}\right)$ to be the transformation $z \rightarrow z+1$. Since $u(z+1)=u(z)$ if and only if $u$ is constant we see that $\operatorname{dim} V_{k}=2 q-2$, and this is equal to $-2 \tilde{v}_{0}\left(p_{k}\right)$.

5.3. Following Weil [8] we introduce notations $R_{0}=1$ and

$$
\begin{aligned}
& R_{j}=a_{1} b_{1} a_{1}^{-1} b_{1}^{-1} \ldots a_{j} b_{j} a_{j}^{-1} b_{j}^{-1} \\
& R_{g+k}=R_{g} c_{1} \ldots c_{k} \\
& \bar{a}_{j}=R_{j-1} b_{j}^{-1} R_{j}^{-1} \\
& \bar{b}_{j}=R_{j} a_{j}^{-1} R_{j-1}^{-1} \\
& \bar{c}_{k}=R_{g+k} c_{k} R_{g+k}^{-1} \quad(1 \leqslant j \leqslant g, 1 \leqslant k \leqslant n) .
\end{aligned}
$$


It is easy to check that $\bar{a}_{y}, \bar{b}_{j}, \bar{c}_{k}$ are generators of $\pi_{i}$. Moreover, the condition $h(w)=0$ can be written in the form

$$
\sum_{j=1}^{g} h\left(a_{j}\right) a_{j}^{-1} R_{j}^{-1}\left(1-\bar{a}_{j}\right)+\sum_{j=1}^{g} h\left(b_{j}\right) b_{j}^{-1} R_{j}^{-1}\left(\bar{b}_{j}-1\right)+\sum_{k=1}^{n} h\left(c_{k}\right) R_{g+k}^{-1}=0 .
$$

We regard the left-hand side of (8) as a mapping $F: V^{2 g} \times V_{1} \times \ldots \times V_{n} \rightarrow V$. Our task is to find the dimension of the nullspace of $F$.

It will be proved below that $F$ is surjective, and also that the number of linearly independent coboundaries is $2 q-1$. From this it follows that the nullspace of $F$ has dimension $2 g(2 q-1)-2 \sum \tilde{v}_{0}(p)-(2 q-1)$, and that the dimension of $P H\left(\pi_{i}\right)$ is $2(g-1)(2 q-1)-$ $2 \sum \tilde{v}_{0}(p)=2 d_{i}$, by comparison with (3). To find the dimension of $H\left(\pi_{i}\right)$ we merely replace $V_{k}$ by $V$ whenever $r_{k}=\infty$. The dimension increases by one for each change, in agreement with the statement of Lemma 4.

5.4. We shall now show that $F$ is surjective. Suppose that this is not so. Then there exists a nonzero linear functional $v^{\prime}$ on $V$ that vanishes on all the subspaces $V \cdot\left(\bar{a}_{j}-1\right)$, $V \cdot\left(\bar{b}_{j}-1\right)$ and $V \cdot\left(c_{k}-1\right) R_{g+k}^{-1}=V \cdot R_{g+k}^{-1}\left(\bar{c}_{k}-1\right)=V \cdot\left(\bar{c}_{k}-1\right)$. If $v^{\prime}$ annihilates $v \cdot(a-1)$ and $v \cdot(b-1)$ for all $v \in V$, it also annihilates $v \cdot(a b-1)=v \cdot a \cdot(b-1)+v \cdot(a-1)$. Since $\left\{\bar{a}_{j}, \bar{b}_{j}, \bar{c}_{k}\right\}$ is a system of generators it follows that $v^{\prime}$ annihilates $v \cdot(a-1)$ for all $v \in V, a \in \pi_{i}$.

Assume first that there is a loxodromic $\sigma(a), a \in \pi_{i}$; we may take it to be $z \rightarrow \omega z$ where $\omega$ is not a root of unity. Since $z^{m} \cdot a=\omega^{m+1-a} z^{m}$ we have $z^{m} \cdot(a-1)=0$ only for $m=q-1$. Therefore, $v^{\prime}$ must be a multiple of the linear functional that maps $v$ on its middle coefficient. It follows that $v \cdot n-v$ has middle coefficient zero for all $v \in V$ and all $b \in \pi_{i}$. We write $\sigma(b)$ in the form $(\alpha z+\beta) /(\gamma z+\delta)$ and apply the result to $v=z^{2 q-2}$ and $v=1$. In this way we obtain $\alpha \beta=\gamma \delta=0$, and this is true only when $\sigma(b)$ is a multiple of $z$ or $1 / z$. It follows that a pair of points (in our case 0 and $\infty$ ) is left invariant by all transformations in $\Gamma_{i j}$. But then $\Gamma_{i j}$ is an elementary group, and this possibility has been ruled out by Lemma 1.

Consider next the case of a parabolic $\sigma(a)$; we take it to be $z \rightarrow z+1$. It is clear that $v(z+1)-v(z)$ has zero first coefficient, while the others are arbitrary. Therefore $v^{\prime}$ takes $v$ into its first coefficient, and we conclude that $v \cdot b-v$ has first coefficient zero for all $v \in V, b \in \pi_{i}$. We apply this to $v=z^{2 \alpha-2}$ and $v=1$. With the same notation as above we obtain $\alpha^{2 q-2}=1, \gamma=0$. Hence $\sigma(b)$ is a euclidean motion. But a discrete group of euclidean motions is elementary, and we have again reached a contradiction.

There cannot be only elliptic transformations $\sigma(a)$, for in that case $\Gamma_{i j}$ would be a finite group, and consequently elementary.

To complete the proof of the lemma we must still show that there are exactly $2 q-1$ linearly independent coboundaries, and this will be so if $v \cdot a-v=0$ for all $a \in \pi_{\mathfrak{i}}$ implies 
$v=0$. Suppose that $v$ satisfies this condition. If there is a loxodromic $\sigma(a)$, normalized as above, the same computation as before shows that $v$ must be a multiple of $z^{q-1}$. However, with the same notation as before, $z^{q-1} \cdot(b-1)=0$ gives $(\alpha z+\beta)^{q-1} \cdot(\gamma z+\delta)^{q-1}=z^{q-1}$ which is true only if $\alpha \gamma=\beta \delta=0$. This means that the pair 0 and $\infty$ remains invariant, and we have the same contradiction as before. Similarly, if there is a parabolic $\sigma(a)$, chosen as before, the condition $v(z+1)=v(z)$ shows that $v$ is constant, and $1 \cdot(b-1)=0$ leads to $(\gamma z+\delta)^{2 q-2}=1$, hence $\gamma=0, \delta^{2 q_{-2}}=1$. Again, $\sigma(b)$ would be a euclidean motion, and the contradiction is reached. (This part of the proof can be found in Bers [4].)

\section{Integrals of the second kind}

6.1. The integrals of the second kind are of course the elements of $E_{i} / E_{i 1}$. We are presently preparing to prove Theorem 1 .

For typographical reasons we write $d$ instead of $d_{i}$. Let $\varphi_{1}, \ldots, \varphi_{d}$ be a basis of $D_{i}^{q}(0)$. The Wronskian $W=\operatorname{det} \varphi_{k}^{(v)}, k=1, \ldots, d, v=0, \ldots, d-1$ is not identically zero. We choose $\zeta_{0} \in \Omega_{i j}$ with $W\left(\zeta_{0}\right) \neq 0$ (a non-Weierstrass point). With this choice there exists a new basis, which we continue to denote by $\varphi_{1}, \ldots, \varphi_{d}$, with the property that

$$
\varphi_{k}(z)=\left(z-\zeta_{0}\right)^{k-1}+O\left(\left|z-\zeta_{0}\right|\right)
$$

LEM A 5. Every $f \in P E_{i}$ has a unique decomposition $f=f_{0}+f_{1}$, where $f_{0}$ has zero periods and $f_{1}$ is regular in $\Omega_{i}$ as well as over the cusps, except for poles of at most order $d$ at points equivalent to $\zeta_{0}$.

Proof. Let $\alpha_{0}$ and $\alpha$ be the one point divisors determined by the projections of $\zeta_{0}$ and some other point $\zeta \in \Omega_{i j}$. It is clear that $D_{i}^{q}\left(d \alpha_{0}\right)$ reduces to zero, for a linear combination of the $\varphi_{k}$ cannot vanish to the order $d$ at $\zeta_{0}$ without being identically zero. For even stronger reason $D_{i}^{q}\left(m \alpha+d \alpha_{0}\right)=0$ for any integer $m \geqslant 0$. It follows by use of (4) that $\operatorname{dim} D_{i}^{1-q}\left(-m \alpha-d \alpha_{0}\right)=m$. For this reason there exists a differential of order $1-q$ that is regular except for precisely the singularity $(z-\zeta)^{-m}$ at $\zeta$ and a pole of at most order $d$ at $\zeta_{0}$. If $f$ is regular over the cusps, it suffices to subtract from $f$ a linear combination of such differentials to end up with the desired decomposition $f=f_{0}+f_{1}$.

In modified form the same method serves to reduce the singularities over the cusps. We refer for notations to the proof of Lemma 1 (Section 2.2). By assumption $\varphi=\partial^{2 q-1} f$ has a development $\sum c_{k} e^{2 \pi i k z}$ with $c_{0}=0$ and only a finite number of negative $k$. It follows that $f$ itself has a development $f=\sum a_{k} e^{2 \pi i k z}+v(z), v \in V$. The reduced degree of the term $e^{2 \pi i k z}$ in 
the development of a differential of order $1-q$ is $k-(q-1)$. Evidently, our method permits us to eliminate all terms with negative reduced degree. In other words, there exists an $f_{0} \in D^{1-q}$ such that $f_{1}=f-f_{0}=\sum_{k=q-1}^{\infty} b_{k} e^{2 \pi i k z}+v(z)$. It follows that $\partial^{2 q-1} f_{1}$ contains only terms $e^{2 \pi i k z}$ with $k \geqslant q-1$, so that $f_{1}$ is regular over the cusp. This completes the existence part of our proof.

To prove uniqueness we observe that if $f_{0}+f_{1}=0$, then $f_{1}$ is a differential of order $1-q$, and $f_{1} \varphi_{k}$ is a first order differential. The sum of the residues must vanish, and since the only possible residue is at $\zeta_{0}$ it follows that $f_{1}$ is polefree, and hence equal to zero.

The above proof is in essence due to Eichler [6]. It was necessary to repeat it because of the different circumstances.

CoR OLL A R. An Eichler integral which actually has a pole of order less than or equal to $d$ at $\zeta_{0}$, and no other singularities, cannot have the same periods as an integral of the first kind.

This is just another formulation of the uniqueness.

6.2. Proof of Theorem 1. We wish to show that $\operatorname{dim}\left(\operatorname{pd} P E_{i} / \mathrm{pd} E_{i 1}\right)=d$. The preceding lemma makes it clear that the dimension is at most $d$, and the theorem will be proved if we can exhibit an $f_{1}$ with a pole of exactly order $k$ for any $k \leqslant d$.

We do this by explicit construction. It is no restriction to assume that $\infty$ is an ordinary point for $\Gamma$ (neither a limit point nor an elliptic fixed point). Under these circumstances the Poincaré series

$$
g_{1}(z, \zeta)=\sum_{A \in \Gamma}(z-A \zeta)^{-1} A^{\prime}(\zeta)^{q}
$$

converges and represents a meromorphic function of $z$ on all of $\Omega$. For a fixed $B \in \Gamma$ a simple computation yields

$$
g_{1}(B z, \zeta) B^{\prime}(z)^{1-q}-g_{1}(z, \zeta)=\sum_{A \in \Gamma}\left[\left(B^{\prime}(A \zeta) / B^{\prime}(z)^{q-\frac{1}{2}}-1\right](z-A \zeta)^{-1} A^{\prime}(\zeta)^{q}\right.
$$

Because $B^{\prime}(z)$ is of the form $(\gamma z+\delta)^{-2}$, the bracketed expression is a polynomial in $z$ of degree $2 q-1$ which is divisible by $z-A \zeta$. We conclude that $\operatorname{pd}_{B} g_{1}(z, \zeta)$ is a polynomial of degree $2 q-2$, the same in all components of $\Omega_{i}$, and even in all components of $\Omega$. Hence $g_{1}(z, \zeta)$ satisfies the basic requirement for an Eichler integral, and one verifies without difficulty that it is regular over the cusps. Its only singularity is a simple pole at $\zeta$.

To obtain integrals with poles of higher order it suffices to form derivatives with respect to $\zeta$. We set $g_{k}(z, \zeta)=\partial^{k-1} g_{1}(z, \zeta) / \partial \zeta^{k-1}$ and $g_{k}(z)=g_{k}\left(z, \zeta_{0}\right)$. It is clear that $g_{k}$ is an Eichler integral with a pole of order $k$. Theorem 1 is proved.

For later use we record the following observations : 
(A) The functions $g_{k}, k=1, \ldots, d_{i}$, have restrictions $g_{k} \mid \Omega_{i} \in E_{i}$ and $g_{k} \mid \Omega_{j} \in E_{j 1}$ for $j \neq i$. All these restrictions have the same periods, and no integral in $E_{i 1}$ has the same periods as a linear combination of the $g_{k}$.

6.3. We have already used the notation $H(\Gamma) \mid \Gamma_{i j}$. An element of $H(\Gamma) \mid \Gamma_{i j}$ determines a cohomology class on $\pi_{i}$, namely by setting $h(c)=h(\sigma(c))$. This correspondence is obviously injective, and instead of introducing a new notation we shall simply regard $H(\Gamma) \mid \Gamma_{i j}$ as a subspace of $H\left(\pi_{i}\right)$. In the same way $P_{i} H(\Gamma) \mid \Gamma_{i j}$ is a subspace of $P H\left(\pi_{i}\right)$, and the restrictions of pd $P E_{i}$ and pd $E_{i 1}$ can be regarded as lying in $P H\left(\pi_{i}\right)$.

Lemмa 6. There exists a homomorphism $\gamma: \operatorname{pd} P E_{i} \rightarrow P H\left(\pi_{i}\right)$ with kernel pd $E_{i 1}$ whose range is contained in $P_{i} H(\Gamma) \mid \Gamma_{i j}$ and has intersection $\{0\}$ with $\mathrm{pd} E_{i 1} \mid \Gamma_{i j}$.

Proof. An element $h \in \mathrm{pd} P E_{i}$ is, by definition, the cohomology class determined by the periods of some $f \in P E_{i}$. We use Lemma 5 to write $f=f_{0}+f_{1}$. There exists a unique linear combination $g$ of the $g_{k}$ with the same singularity as $f_{1}$. We define $\gamma$ by $\gamma(h)=\operatorname{pd} g \mid \Gamma_{i j}$.

It is clear that $f \in E_{i \mathrm{1}}$ gives $f_{1}=f$ and $g=0, \gamma(h)=0$. Conversely, $\gamma(h)=0$ implies $g=0$, $f_{1} \in E_{i 1}$, and $h=\operatorname{pd} f_{1}$. Hence the kernel is $\operatorname{pd} E_{i 1}$, as asserted. Finally, the corollary to Lemma 5 shows that $\gamma(h) \in \operatorname{pd} E_{i 1} \mid \Gamma_{i j}$ implies $g=0, \gamma(h)=0$.

COROLLARY. The range of $\gamma$ has dimension $d_{i}$.

This is a direct consequence of Lemma 6 together with Theorem 1.

\section{Integrals of the first kind}

7.1. Since $\partial^{2 q-1}$ transforms Eichler integrals into differentials of order $q$ it is natural to try to reverse the process and pass from differentials to integrals through repeated integrations. The difficulty is that this construction may lead to multiple-valued functions, and even if we obtain a single-valued integral on $\Omega_{i j}$, say, it is not certain that it can be extended to an Eichler integral on $\Omega_{i}$. What we can do, however, is to map $D_{i}^{q}(0)$ into $P H\left(\pi_{i}\right)$.

Lемм 7. There is a canonical injection $\delta: D_{i}^{q}(0) \rightarrow P H\left(\pi_{i}\right)$ such that $\delta\left(D_{i}^{q}(0)\right) \cap H(\Gamma) \mid \Gamma_{i j}$ $\subset \mathrm{pd} E_{i 1} \mid \Gamma_{i j}$. Moreover, the inverse image of $H(\Gamma) \mid \Gamma_{i j}$ is contained in $\partial^{2 q-1} E_{i 1}$.

Proof. Given $\varphi \in D_{i}^{q}(0)$ we define $h_{\varphi}$ on $\pi_{i}$ by

$$
h_{\varphi}(c)=\int_{\left(c^{-1} \gamma^{\prime}\right.}(z-\zeta)^{2 q-2} \varphi(\zeta) d \zeta
$$


We recall that $\left(c^{-1}\right)^{\prime}$ is the lift of $c^{-1}$, beginning at $z_{0}$. It is clear that $h_{\varphi}(c) \in V$ and that it depends only on the homotopy class of $c$.

To prove that $h$ is a cocycle we observe that $\left(b^{-1} a^{-1}\right)^{\prime}$, is $\left(b^{-1}\right)^{\prime}$ followed by $B^{-1}\left(a^{-1}\right)^{\prime}$ where $B=\sigma(b)$. Hence,

$$
h_{\varphi}(a b)=\left(\int_{\left(b^{-1}\right)^{\prime}}+\int_{B^{-1}\left(a^{-1}\right)^{\prime}}\right)(z-\zeta)^{2 q-2} \varphi(\zeta) d \zeta
$$

The first integral is $h_{\varphi}(b)$, and a simple calculation shows that the second is $h_{\varphi}(a) \cdot B$. Thus $h_{\varphi}(a b)=h_{\varphi}(a) \cdot b+h_{\varphi}(b)$.

We need to show, moreover, that $h_{\varphi}$ is parabolic. Let $b$ correspond to a loop around a cusp. Then $B=\sigma(b)$ is a parabolic transformation, and as usual we may assume that $B z=z+1$. We can choose the path $b$ in such a way that

$$
h_{\varphi}(b)=\left(\int_{z_{0}}^{z_{0}+i M}+\int_{z_{0}+i M}^{z_{0}-1+i M}-\int_{z_{0}-1}^{z_{0}-1+i M}\right)(z-\zeta)^{2 q-2} \varphi(\zeta) d \zeta
$$

with large $M$. Because $\varphi$ is regular over the cusp it tends to zero as $\operatorname{Im} \zeta \rightarrow \infty$, and hence the middle integral tends to zero as $M \rightarrow \infty$. The convergence of the two other integrals is also assured, and we see at once that $h_{\varphi}(b)$ has the form $v(z+1)-v(z), v \in V$.

Suppose now that $h$ is a coboundary, $h_{q}(c)=v \cdot \sigma(c)-v$ for all $c \in \pi_{i}$. Then, first of all, $h_{p}(c)=0$ when $\sigma(c)$ is the identity, and therefore

$$
f_{\varphi}(z)=\int_{z_{0}}^{z}(z-\zeta)^{2 a-2} \varphi(\zeta) d \zeta
$$

is independent of the path in $\Omega_{i j}$. One verifies by computation that $f_{\varphi} \cdot \sigma(c)-f_{\varphi}=h_{\varphi}(c)=$ $v \cdot \sigma(c) \rightarrow v$. Hence $f_{\varphi}-v$ is invariant, and it follows that $f_{\varphi}=v$ and $\varphi=\partial^{2} \alpha-1 f=0$. We have proved that the mapping which takes $\varphi$ into the cohomology class of $h$ is injective.

To complete the proof of Lemma 7 , assume that $\delta(\varphi) \in H(\Gamma) \mid \Gamma_{i j}$, which means that $h_{\varphi}(c)=h(\sigma(c))$ for some cocycle $h$ on $\Gamma$. It follows as above that (11) is independent of the path, so that $f_{\varphi}$ is again well defined. Moreover, its periods in $\Omega_{i j}$ are given by $h$, and by assumption $h$ has an extension to all of $\Gamma$. We conclude by Lemma 3 that $f_{\varphi}$ can be extended to an Eichler integral on $\Omega_{i}$. Since $f_{\varphi}$ is regular, so is the extension. We have shown that $\delta(\varphi) \in \mathbf{p d} E_{i 1} \mid \Gamma_{i j}$. Also, $\varphi=\partial^{2 a-1} f_{\varphi} \in \partial^{2 q-1} E_{i 1}$. The proof is complete.

7.2. The following observation is a direct consequence of the preceding lemmas.

LEM $\mathrm{M}$ A 8 . The ranges of $\gamma$ and $\delta$ span all of $P H\left(\pi_{i}\right)$.

Since both ranges have dimension $d_{i}$ and $P H\left(\pi_{i}\right)$ has dimension $2 d_{i}$ (Lemma 4 ) this is equivalent to saying that the intersection of the ranges is zero. Suppose that $\delta(\varphi)=\gamma(\operatorname{pd} f)$ 
with $\varphi \in D_{i}^{q}(0), f \in P E_{i}$. Since $\gamma(\operatorname{pd} f) \in P_{i} H(\Gamma)\left|\Gamma_{i j} \subset H(F)\right| \Gamma_{i j}$ it follows by Lemma 7 that $\delta(\varphi) \in \operatorname{pd} E_{i 1} \mid \Gamma_{i j}$, and by Lemma 6 that $\delta(\varphi)=\mathbf{0}$.

7.3. Proof of Theorem 2 (second part). Given $h \in P_{i} H(\Gamma)$ we consider its restriction $h \mid \Gamma_{i j}$ as an element of $P H\left(\pi_{i}\right)$. According to Lemma 8 it has a representation $h \mid \Gamma_{i j}=$ $\gamma\left(h_{1}\right)+\delta(\varphi)$ with $h_{1} \in \operatorname{pd} P E_{i}$ and $\varphi \in D_{i}^{q}(0)$. Since $h \mid \Gamma_{i j}$ and $\gamma\left(h_{1}\right)$ are in $H(\Gamma) \mid \Gamma_{i j}$, so is $\delta(\varphi)$. It follows by Lemma 7 that $\delta(\varphi) \in \operatorname{pd} E_{i 1} \mid \Gamma_{i j}$. Hence $h\left|\Gamma_{i j} \in \operatorname{pd} E_{i 1}\right| \Gamma_{i j}$, and Lemma 3 shows that $h \in \mathrm{pd} P E_{i}$. The opposite inclusion, pd $P E_{i} \subset P_{i} H(\Gamma)$ has already been proved (Lemma 1).

7.4. In order to prove the first part of Theorem 2 it is necessary to take into account differentials with simple poles at the cusps. We shall denote by $\chi_{i}$ the cusp divisor with coefficient -1 at each cusp of $S_{i}$. Accordingly, $D_{i}^{q}\left(\chi_{i}\right)$ will be the space of differentials with at most simple poles over the cusps. We shall write $E_{i}\left(\chi_{i}\right)$ for the space of Eichler integrals $f$ with $\partial^{2 \alpha-1} f \in D_{i}^{q}\left(\chi_{i}\right)$.

We need stronger versions of Lemmas 6-8.

Lемма $6^{\prime} \cdot \gamma\left(\operatorname{pd} P E_{i}\right) \cap \operatorname{pd} E_{i}\left(\chi_{i}\right) \mid \Gamma_{i j}=0$.

Proof. We must show that $g$, as defined in the proof of Lemma 6, cannot have the same periods as $f \in E_{i}\left(\chi_{i}\right)$. If this were so $(g-f) \varphi_{k}$ would be a first order differential, and the sum of the residues would have to vanish. The conclusion $g=0$ will follow if we can show that $f \varphi_{k}$ has zero residues at the cusps.

As usual we assume that the cusp corresponds to $B z=z+1$, and we use $Z=e^{2 \pi i z}$ as local variable. The development of $\partial^{2 q-1} f$ in powers of $Z$ contains no negative powers, but the constant term may be present. Repeated integration shows that $f$ itself can be developed in positive powers of $Z$ plus a polynomial in $z$. Since $f \varphi_{k}$ and $\varphi_{k}$ are both periodic, so is $f$, and the polynomial reduces to a constant. The development of $\varphi_{k}$ begins with a positive power, and the same is consequently true of $f \varphi_{k}$. Hence $f \varphi_{k}$ is regular over the cusp, and the residue is zero.

LEмм а $7^{\prime}$. The homomorphism $\delta$ can be extended to an injection $\delta^{\prime}: D_{i}^{q}\left(\chi_{i}\right) \rightarrow H(\Gamma) \mid \Gamma_{i j} \subset$ $H\left(\pi_{i}\right)$ such that $\delta^{\prime}\left(D_{i}^{a}\left(\chi_{i}\right)\right) \cap H(\Gamma)\left|\Gamma_{i j} \subset \operatorname{pd} E_{i}\left(\chi_{i}\right)\right| \Gamma_{i j}$.

Proof. We can still define $h_{\varphi}$ by (10). It is a cocycle, but no longer parabolic. If $h$ is a coboundary, $h_{\varphi}(c)=v \cdot \varphi(c)-v$, it follows as before that $f_{\varphi}(z)$, defined by (11), is independent of the path, and that $f_{\varphi}-v$ is invariant. If the cusp is chosen as before, $\varphi(\zeta)$ remains bounded as $\operatorname{Im} \zeta \rightarrow+\infty$. Therefore $f_{p}-v$ grows like a polynomial in $z$, but because the function is periodic only the constant term can be different from zero. This means that $f_{\varphi}-v$ is regular 
over the cusp when regarded as a differential of order $1-q$, and we conclude that $f_{\varphi}=v$. The rest of the proof is exactly as before.

LEMMA $8^{\prime}$. The ranges of $\gamma$ and $\delta^{\prime}$ span all of $H\left(\pi_{i}\right)$.

As previously, this follows from Lemmas $6^{\prime}$ and $7^{\prime}$ together with the fact that

$$
\operatorname{dim}\left(\operatorname{pd} P E_{i}\right)=d_{i}, \operatorname{dim} \delta^{\prime}\left(D_{i}^{q}\left(\chi_{i}\right)\right)=d_{i}+n_{i} \text { and } \operatorname{dim} H\left(\pi_{i}\right)=2 d_{i}+n_{i} .
$$

The first part of Theorem 2 can now be proved by repeating the reasoning in Section 7.2 almost word by word.

\section{Proof of Theorem 3}

8.1. We recall that $\Omega_{I}$ is a finite union of sets $\Omega_{i}, i \in I$. The space $P E_{1}$ consists of functions $f$ on $\Omega_{I}$ whose restrictions $f^{(i)}=f \mid \Omega_{i}$ for $i \in I$ belong to $P E_{i}$ and have the same periods.

Our first observation is that Lemma 5 generalizes to $f \in P E_{I}$. For that purpose we must first choose non-Weierstrass points $\zeta_{0}^{(i)} \in \Omega_{i}$. The individual decompositions $f^{(i)}=$ $f_{0}^{(i)}+f_{1}^{(i)}$ combine to a decomposition $f=f_{0}+f_{1}$ where $f_{1}$ belongs to $P E_{I}$ and is regular except for poles of order $\leqslant d_{i}$ at the points $\zeta_{0}^{(t)}$. We know moreover that $\operatorname{pd} f=\operatorname{pd} f_{1}$ belongs to $\operatorname{pd} E_{I 1}$ if and only if $f_{1}$ is polefree. Counting the possible singularities we deduce at once that $\operatorname{dim}\left(\operatorname{pd} E_{I} / \mathrm{pd} E_{t 1}\right) \leqslant \sum_{i \in I} d_{i}=d_{I}$.

To prove the opposite inequality we need only consider the functions $g_{k}$ introduced in Section 6.2. For identification purposes the functions with pole at $\zeta_{0}^{(i)}$ will now be denoted by $g_{k}^{(i)}, 1 \leqslant k \leqslant d_{i}$. They are defined in $\Omega_{I}$, and our remark (A) shows that they belong to $P E_{I}$. Their total number is $d_{I}$, and the periods pd $g_{k}^{(i)}$ are linearly independent modulo pd $P E_{I 1}$. Indeed, suppose that $\sum_{i, k} c_{i k} \operatorname{pd} g_{k}^{(i)}=\operatorname{pd} f$ with $f \in P E_{I 1}$. By restriction to $\Omega_{i}$ we find that $\sum_{k} c_{i k} \operatorname{pd} g_{k}^{(i)}=\operatorname{pd} f$ with $f \in P E_{i 1}$, and according to (A) this is possible only when $c_{i k}=0$. The theorem is proved.

It has already been remarked that Theorem 4 requires no proof.

Remark. When this paper was already written I was informed by Dr. Irwin Kra that he too has investigated $H(\Gamma)$ in its relationship to the differentials and Eichler integrals on an invariant part of $\Omega$. Because he uses the methods of Bers where $I$ use those of Eichler there seems to be no direct overlap between his work and mine. It is likely, however, that the two points of view will eventually complement each other and lead to a fuller understanding of the whole circle of ideas. 


\section{References}

[1]. Ahlfors, L. V., Finitely generated Kleinian groups. Amer. J. Math., 86 (1964) 413-429 and 87 (1965), 759 .

[2]. —— Eichler integrals and Bers' area theorem. Michigan Math. J., 15 (1968), 257-263.

[3]. BERs, L., A non-standard integral equation with applications to quasiconformal mappings. Acta Math., 116 (1966), 113-134.

[4]. - Inequalities for finitely generated Kleinian groups. J. Analyse Math., 18 (1967), $23-41$.

[5]. —- On Ahlfors' finiteness theorem. Amer. J. Math., 89 (1967), 1078-1082.

[6]. ErChLER, M., Eine Verallgemeinerung der Abelschen Integrale. Math.Z., 67 (1967), 267-278.

[7]. Greenserg, L., On a theorem by Ahlfors and conjugate subgroups of Kleinian groups. Amer. J. Math., 89 (1967), 56-68.

[8]. WEIL, A., Remarks on the cohomology of groups. Ann. of Math., 80 (1964), 149-157.

Received August 1, 1968 